\title{
Búsqueda de la mutación delta F508 y análisis de dos polimorfismos de nucleótido único en el gen CFTR, en una muestra de población general de Valparaíso, Chile
}

\author{
Alejandra Vera $\mathrm{L}^{1 \mathrm{a}}, \mathrm{C}$ Carlos $\mathrm{F}$ Henríquez-Roldán ${ }^{2 b}$, \\ Francisco J González $\mathbf{R}^{1 \mathrm{lc}}$, G raciela Molina $F^{1 d}$. \\ Screening of the delta-F508 mutation and \\ analysis of two single nucleotide \\ polymorphism of the CFTR gene, \\ in a sample of the general population of \\ Valparaíso, Chile
}

Background: The Cystic Fibrosis (CF) carrier rate in Chile was estimated to be 1/40. CF is caused by mutations in the Cystic Fibrosis Transmembrane Conductance Regulator (CFTR) gene. Delta F508 mutation is the most common in CF patients in Chile and worldwide. Delta F508 has linkage disequilibrium with two Single Nucleotide Polymorphisms (SNP), often used to define the haplotypic frameworks of CF mutations. Aim: To know the frequency of the delta F508 mutation and to establish the SNPs, M470V and T854T, haplotypic frequency, in a Valparaiso general population sample. Subjects and Methods: Fifty subjects were studied. Genetic material was obtained from blood samples, amplified by PCR and analyzed by restriction fragment length polymorphism. Results: Two of the 100 chromosomes analyzed, carried the delta F508 mutation. Therefore, the observed frequency carrier rate (0.02) was higher than the expected (0.01). Both carrier chromosomes had the same SNPs haplotypic framework (1-2). In normal chromosomes, the haplotype 2-1 was the most common. Discussion: These results suggest that the chromosomes that bear delta F508 mutation have most likely a Mediterranean European origin, since this haplotypic framework has been reported in that region. We suggest that CF could be more common in Valparaiso than it was previously estimated (Rev Méd Chile 2005; 133: 767-75).

(Key Words: Cystic fibrosis; Cystic fibrosis transmembrane conductance regulator; Delta F508-CFTR protein)

\footnotetext{
Recibido el 31 de agosto, 2004. Aceptado en versión corregida el 30 de marzo, 2005.

Trabajo financiado por los proyectos DIPUV 20/2000 y 28/2001.

${ }^{1}$ Laboratorio de Biología y Genética Molecular, Centro de Investigaciones Médicas, Escuela de Medicina, Facultad de Medicina. ${ }^{2}$ Departamento de Estadística, Instituto de Matemática y Física, Facultad de Ciencias, Universidad de Valparaíso.

aBiólogo

${ }^{b}$ Doctor en Bioestadística

'Licenciado en Biología, Doctor en Ciencias, con mención en Biología Molecular.

dMédico, Doctor en Ciencias Biomédicas.
}

Correspondencia a: Dra. Graciela Molina F. Laboratorio de Biología y

Genética Molecular. Hontaneda 2654, Casilla 92-V, Valparaíso. Fono:

56-32-507322. Fax: 56-32-507321. E-mail: graciela.molina@uv.cl 
工 Fibrosis Quística (FQ), (MIM 219700) ${ }^{1}$ es causada por mutaciones presentes en su gen, denominado Cystic Fibrosis Transmembrane Conductance Regulator (CFTR), (MIM 602421) ${ }^{1}$. Este gen codifica para una proteína de membrana llamada regulador de la conductancia transmembrana en la fibrosis quística (proteína CFTR) ${ }^{2}$. Su función principal es regular el flujo de electrolitos de cloro y, secundariamente, el flujo de sodio y agua hacia el espacio extracelular ${ }^{3}$. La disfunción del CFTR causa la obstrucción, principalmente, de las vías aéreas y los ductos pancreáticos ${ }^{4}$.

La mutación causante de FQ más frecuente a escala mundial (67\% de los casos registrados), corresponde a la denominada delta F508 e identifica la deleción del triplete CTT en la región codificante (exón 10) del gen $\mathrm{CFTR}^{2,5,6}$. Esta mutación se traduce en la ausencia del aminoácido fenilalanina en la posición 508 del polipéptido normal ${ }^{2,6}$. Actualmente, se han descrito más de 1.000 mutaciones en el gen CFTR causantes de FQ ${ }^{7}$.

La incidencia de la FQ está determinada por la composición étnica de cada población $n^{5}$. En Chile, sobre la base de nuestra composición étnica mixta (mezcla caucásico-amerindia ${ }^{8}$ ), se estimó una incidencia de FQ de 1:4.000 nacidos vivos ${ }^{9}$. El número de casos esperados, basándose en la certeza de esta incidencia, comparado al número de casos registrados, indica que existiría un subdiagnóstico mayor a $50 \%{ }^{10}$.

Recientemente se ha observado que algunos polimorfismos de nucleótido único (Single Nucleotide Polymorphism); (SNP) presentes en el gen CFTR, pueden influir en el grado de severidad de esta enfermedad ${ }^{11-13}$. Adicionalmente, son de gran utilidad para definir el marco haplotípico de las mutaciones causantes de $\mathrm{FQ}^{14,15}$ y para estudiar la evolución del locus CFTR en una población ${ }^{16-18}$, debido a que presentan una tasa de mutación muy baja (10-9 mutaciones por generación $)^{19}$.

Los objetivos de este estudio son: determinar la incidencia de la mutación delta F508 y establecer las frecuencias de los haplotipos de dos polimorfismos de nucleótido simple (SNPs), en población general de la ciudad de Valparaíso.

La mutación delta F508 está presente en 40\%$50 \%$ de los cromosomas de los pacientes FQ chilenos $^{20-22}$. Considerando esta frecuencia y la incidencia calculada de FQ en Chile, se esperaba encontrar un cromosoma portador de esta muta- ción en la muestra ( $p=0,0125)$, con un intervalo de confianza de 87,5\% $(Z=1,145 p=0,1251)^{23}$.

Adicionalmente, se analizaron dos SNPs exónicos dialélicos. Estos corresponden a T854T sustitución silente (T/G 2.694) en el exón 14a y M470V, que identifica una sustitución aminoacídica en el exón 10 del gen $\mathrm{CFTR}^{24}$. Las frecuencias haplotípicas en esta muestra de población mixta chilena, permitirán estructurar una base de datos de un grupo control, para establecer comparaciones con pacientes que presenten formas típicas y atípicas de FQ. Debido a la baja tasa mutacional de los SNPs analizados, con respecto a los haplotipos a estudiar, no se espera observar gran diferencia o variación con respecto a lo que se ha descrito para países europeos ${ }^{14,16,25}$.

\section{MATERIALES Y MÉTODOS}

Sujetos. Fueron seleccionados 50 individuos (100 cromosomas) normales, de un total de 229 escolares de octavo año básico de distintos estratos sociales, los cuales habían sido muestreados previamente para otro estudio (proyecto DIPUV 20/ 2000). El muestreo de los 229 escolares se realizó incluyendo a todos los colegios y escuelas de la comuna de Valparaíso durante los años 2001 y 2002. En aquellos establecimientos que aceptaron participar, se solicitaron voluntarios informados. El número de individuos muestreados fue dependiente de la proporción que el tipo de establecimiento representaba en la totalidad del universo de la comuna (municipalizados, subvencionados, y particulares). Los autores no introdujeron ningún tipo de sesgo en su selección. Todos los individuos que participaron en este estudio, contaban con un consentimiento informado de sus padres o representantes legales. En los 229 individuos se mantuvo la representatividad por tipo de establecimiento. La muestra corresponde al 8,6\% de la matrícula total promedio de los dos años (2.770 y 2.553, respectivamente).

Manteniendo la representatividad de la distribución por sexo y tipo de colegio, los cincuenta individuos fueron seleccionados al azar.

Análisis genético molecular. El material genético fue extraído de muestras de sangre mediante el método descrito por Miller y Dykes ${ }^{26}$. 
La mutación delta F508 fue detectada por amplificación mediante mutagénesis sitio dirigida, mediada por la PCR del exón 10 del gen CFTR $^{27}$. Esta técnica permite crear un sitio de restricción alelo específico para la enzima MboI. Para la amplificación se utilizaron las condiciones y los partidores descritos por Friedman et $\mathrm{al}^{27}$. En un volumen de reacción de $50 \mu \mathrm{l}$ se agregan $10 \mathrm{ng}$ de ADN genómico, 0,5 $\mu \mathrm{M}$ de cada partidor, $0,2 \mathrm{mM}$ dNTPs, $1,5 \mathrm{mM} \mathrm{MgCl} 2$, buffer Taq $1 \mathrm{X}$ y 1,25 unidades de Taq polimerasa (Promega ${ }^{\circledR}$ ). La identificación de los alelos se realizó mediante la técnica de análisis del polimorfismo del tamaño de los fragmentos de restricción (Restriction Fragment Length Polymorphism, RFLP). Los productos amplificados fueron digeridos con la enzima correspondiente. Los fragmentos resultantes se resolvieron mediante electroforesis denaturante $(7 \mathrm{M}$ úrea), en un gel de poliacrilamida al $6 \%$, el cual posteriormente fue teñido utilizando $\mathrm{AgNo}_{3}$ 0,2\%.

Los polimorfismos de nucleótido único también fueron analizados mediante la técnica de
RFLP. Los exones 10 y 14a del gen CFTR fueron amplificados utilizando los partidores y condiciones descritos por Zielenski et $\mathrm{al}^{24}$. Los productos amplificados fueron digeridos con la enzima de restricción apropiada y luego, los fragmentos resultantes, fueron separados mediante electroforesis en geles de agarosa al 2\%, teñidos con bromuro de etidio y visualizados a una longitud de onda $(\lambda)$ de $302 \mathrm{~nm}$ en un transiluminador UV (2UV ${ }^{\mathrm{TM}} \mathrm{LM}-20 \mathrm{E}$ UVP).

Los tamaños de los fragmentos obtenidos en la amplificación y análisis por RFLP de la mutación delta F508 se muestra en la Figura 1 y de los SNPs, en la Tabla 1.

Estadística. Las frecuencias alélicas y genotípicas de la mutación delta F508 y de los SNPs se obtuvieron por conteo directo. Las frecuencias genotípicas esperadas se calcularon utilizando el algoritmo de Levene ${ }^{28}$ con el programa PopGene, y la posible desviación del equilibrio de Hardy-Weinberg se evaluó mediante el test de Chi cuadrado $\left(\chi^{2}\right)$ y tasa
FIguRA 1. Esquema del análisis de la mutación delta F508. Se muestran los tamaños de los fragmentos amplificados en pares de bases $(\mathrm{pb})$, enzimas de restricción utilizadas y tamaños de los fragmentos resultantes de la digestión enzimática para el análisis por RFLP para la secuencia normal y mutada.

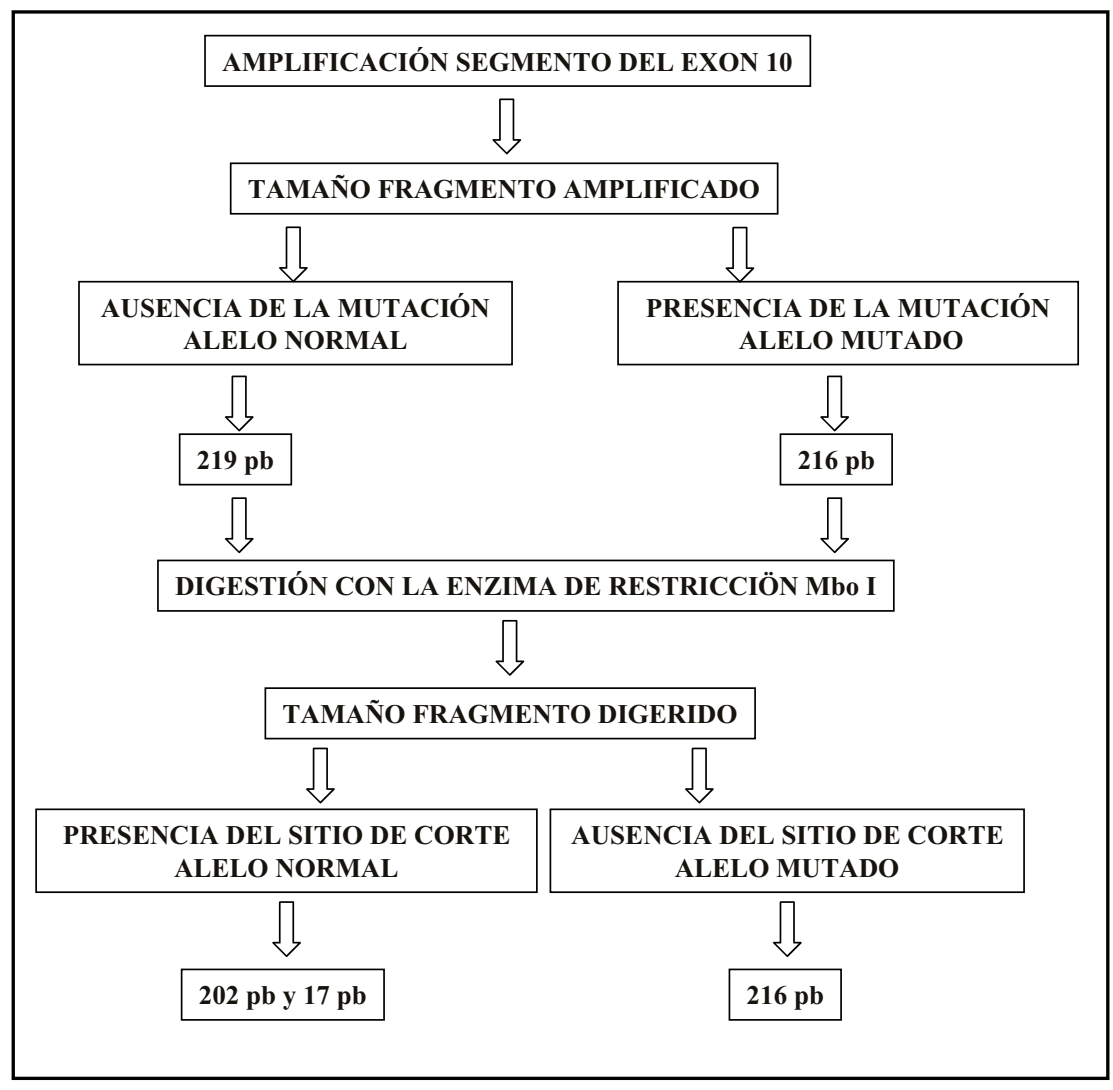


Tabla 1. Análisis de los polimorfismos de nucleótido único (SN Ps). U bicación dentro del gen, tamaños amplificados, enzimas utilizadas para el RFLP, tamaño de los fragmentos resultantes de la digestión, nucleótido presente en el sitio polimórfico y simbología de cada alelo (Ref. 24).

\begin{tabular}{|c|c|c|c|c|c|c|c|}
\hline SNP & Ubicación & $\begin{array}{c}\text { Tamaño* } \\
\text { fragmento } \\
\text { amplificado }\end{array}$ & $\begin{array}{l}\text { Enzima de } \\
\text { restricción } \\
\text { utilizada }\end{array}$ & $\begin{array}{c}\text { Nucleótido } \\
\text { presente } \\
\text { en el sitio }\end{array}$ & $\begin{array}{l}\text { Sitio de } \\
\text { corte }\end{array}$ & $\begin{array}{c}\text { Tamaños* } \\
\text { fragmentos } \\
\text { digeridos }\end{array}$ & $\begin{array}{l}\text { Símbolo } \\
\text { del alelo }\end{array}$ \\
\hline \multirow[t]{3}{*}{ M470V } & \multirow{3}{*}{$\begin{array}{c}\text { Exón } 10 \\
\text { Nucleótido } \\
+1540 \\
\text { Exón 14a }\end{array}$} & \multirow[t]{2}{*}{492} & \multirow{2}{*}{ Hph I } & Adenina & Ausente & 492 & 1 (M470) \\
\hline & & & & Guanina & Presente & 310 y 191 & 2 (V470) \\
\hline & & \multirow{3}{*}{511} & \multirow{3}{*}{ Ava II } & Timina & Ausente & 511 & 1 \\
\hline \multirow[t]{2}{*}{ T854T } & Nucleótido & & & & & & (Ava II -) \\
\hline & & & & Guanina & Presente & 386 y 125 & $\begin{array}{c}2 \\
\text { (Ava II +) }\end{array}$ \\
\hline
\end{tabular}

*Tamaño en pares de bases.

de probabilidades $\left(\mathrm{G}^{2}\right)$. Los haplotipos de los individuos que resultaron ser heterocigotos para las variantes alélicas de los dos SNPs analizados fueron reconstruidos mediante inferencia Bayesiana utilizando el programa Haplotyper ${ }^{29}$.

\section{RESULTADOs}

I. Búsqueda de la mutación delta F508. El análisis de 100 cromosomas de individuos normales sanos de la ciudad de Valparaíso, permitió detectar dos individuos portadores heterocigotos para esta mutación. En la Figura 2 se muestra una electroforesis denaturante en gel de poliacrilamida. En los individuos 2 y 8 , se distinguen dos bandas. La banda superior tiene un tamaño de 216 pb e identifica al producto amplificado del cromosoma que presenta la mutación delta F508. En este alelo, el sitio de restricción para la enzima Mbo I está ausente y, debido a que posee una deleción de 3 pb, presenta un tamaño menor al amplificado del control normal no digerido (219 pb). La banda inferior, de $202 \mathrm{pb}$, es del mismo tamaño que los dos controles normales digeridos y que corresponde al alelo normal. La presencia de los dos portadores heterocigotos, fue confirmada efectuando todo el proceso por duplicado desde la amplificación. La digestión parcial se descartó mediante controles externos y redigestión del producto de PCR.
La frecuencia de delta F508 en los 50 individuos analizados, correspondió a 0,04 (4\%, varianza $\pm 1,92 \times 10^{-4}$ ). El intervalo de confianza para esta frecuencia es de 85,77\% ( $Z=1,066, p=0,1423)$. Este locus no presenta desviación del equilibrio de Hardy-Weinberg $\left(\chi^{2}=0,010309, \mathrm{p}>0,9 ; \mathrm{G}^{2}=\right.$ 0,020410, $\mathrm{p}>0,8$ ).

II. Determinación de los alelos, genotipos y frecuencias de los SNPs: M470V y T854T. Los alelos de cada SNP se identifican según el tamaño en pares de bases (pb) que presenten los fragmentos resultantes de la digestión enzimática (Tabla 1). Los genotipos de ambos SNPs se observan en la Figura 3. En ésta, se observan individuos representativos de cada genotipo: individuos homocigotos para las variables alélicas de cada SNP e individuos heterocigotos para las mismas. En la Figura 3A, se observan las distintas variantes alélicas del SNP T854T. En el carril 3 se observa un fragmento de $511 \mathrm{pb}$. Este identifica el alelo 1, que no posee el sitio de corte para la enzima de restricción Ava II (Ava II -). En el caso de que el sitio de corte para dicha enzima esté presente, se producen dos fragmentos de $386 \mathrm{pb}$ y 125 pb como resultado de la digestión enzimática. En el carril 4 se observa el fragmento de $386 \mathrm{pb}$ que identifica el alelo 2 (Ava II +). En la Figura 3B, se puede observar las variantes alélicas del SNP M470V. En el carril 3, se 
distinguen dos bandas, la mayor de $301 \mathrm{pb}$ y la menor de $191 \mathrm{pb}$, que identifican al alelo 2 (V470). Estos fragmentos son el producto de la digestión de la enzima Hph I. En el carril 4 se observa una banda de $492 \mathrm{pb}$, correspondiente al alelo 1 (variante M470), esta variante alélica no presenta sitio de restricción para la enzima Hph I; por lo tanto, el fragmento es del mismo tamaño que el producto amplificado no digerido.

Los alelos y genotipos fueron determinados en los 50 individuos. Las frecuencias alélicas de

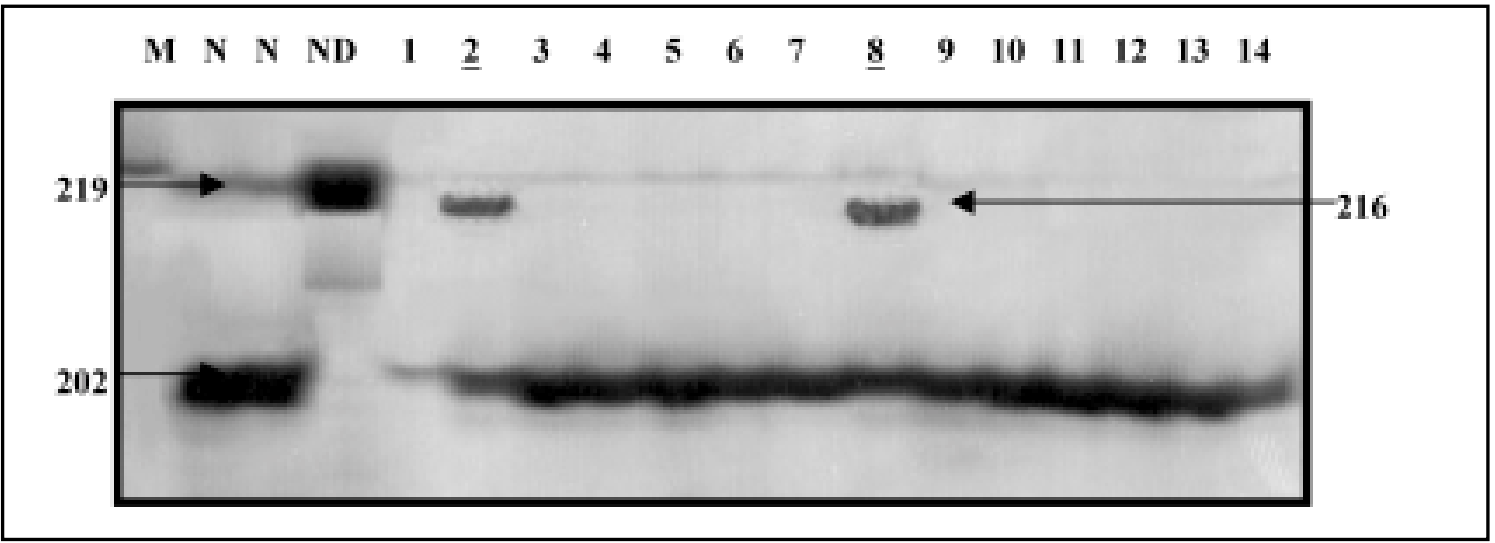

Figura 2. Detección de la mutación delta F508. Electroforesis en gel de poliacrilamida denaturante al $6 \%$, teñido con plata. Visualización de los productos resultantes de la digestión con la enzima de restricción Mbo I de los amplificados para un segmento del exón 10. (M) marcador de peso molecular (pGEM ${ }^{\circledR} \mathrm{ADN}$ Markers); (N) controles normales digeridos (202 pb); (ND) control normal no digerido (219 pb). Los carriles $1 \mathrm{al} \mathrm{14,} \mathrm{muestra}$ 14 individuos analizados. En los carriles 2 y 8 (subrayados) se muestran los dos individuos portadores heterocigotos de la mutación. La banda de 216 pb corresponde al alelo mutado.

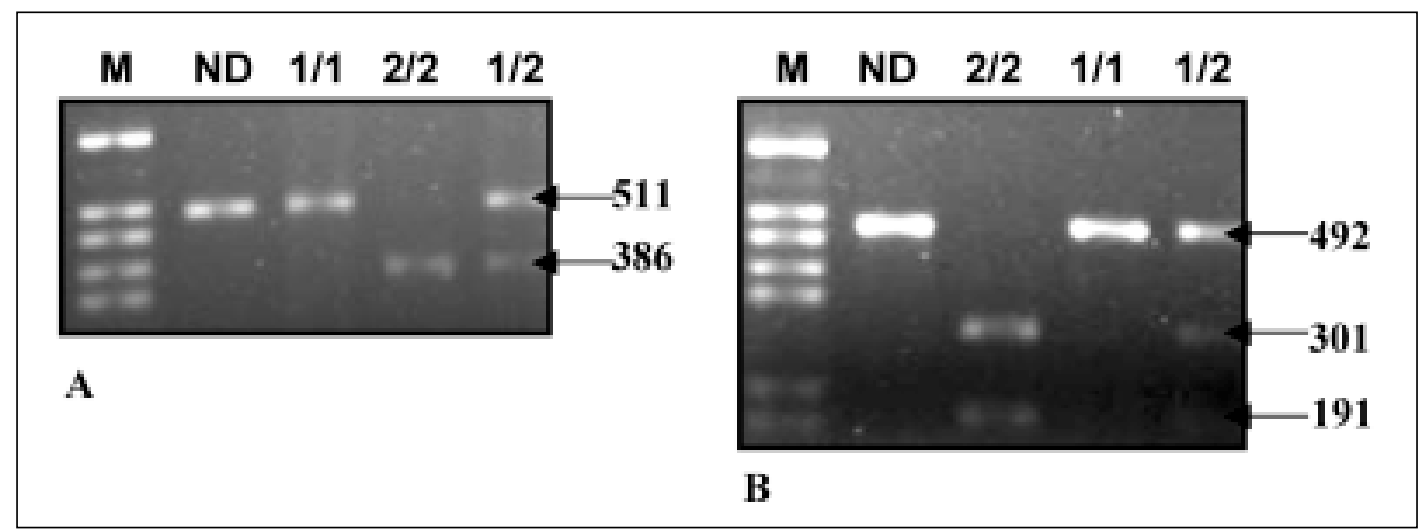

Figura 3. Análisis de los genotipos de los SNPs M470V y T854T. 3A. Electroforesis en gel de agarosa al 2\% de los productos resultantes de la digestión enzimática de fragmentos amplificados del exón 14a con la enzima Ava II para el análisis de T854T. 1; alelo 1 (Ava II-); 2; alelo 2 (Ava II+). 3B. Electroforesis en gel de agarosa al $2 \%$ de los productos resultantes de la digestión enzimática de fragmentos amplificados del exón 10 con la enzima Hph I para el análisis de M470V. 1, alelo 1 (M470); 2, alelo 2 (V470). (M) marcador de peso molecular pGEM ${ }^{\circledR}$ DNA Markers; (ND) producto amplificado no digerido. 
ambos SNP se muestran en la Tabla 2. Los alelos más frecuentes en los individuos analizados correspondieron a $2(54 \%)$ y 1 (62\%). Para el SNP M470V, el genotipo 1/2 es el que se observa con mayor frecuencia, mientras que los dos genotipos restantes presentan frecuencias menores. En el caso de T854T, el genotipo 1/2 presentó la mayor frecuencia observada, mientras que el genotipo 2/ 2 fue el más escaso entre los individuos analizados. Estas frecuencias se muestran en la Tabla 2. No se observó desviación del equilibrio de HardyWeinberg para el locus M470V $\left(\chi^{2}=0,774460, p\right.$ $\left.>0,3 ; \mathrm{G}^{2}=0,775050, \mathrm{p}>0,3\right)$, ni para el locus T854T $\left(\chi^{2}=1,302762, p>0,2 ; G^{2}=1,291850, p>0,2\right)$.

III. Análisis de haplotipos. El haplotipo 2-1 fue el más común entre los cromosomas estudiados, mientras que el haplotipo 2-2, fue el más escaso. Los individuos que portan la mutación delta F508 presentaron distintos genotipos para ambos SNPs. Uno de ellos es heterocigoto $(1 / 2 ; 1 / 2)$ tanto para las variantes alélicas M470V como para T854T, y el otro es homocigoto para ambos SNPs $(1 / 1 ; 2 / 2)$. Sin embargo, la reconstrucción de los haplotipos de ambos cromosomas portadores de delta F508 indican el mismo marco haplotípico para los SNPs, que corresponde al 1-2. Este haplotipo presentó también la segunda mayoría entre los cromosomas no portadores (Tabla 2).

\section{DisCUSIÓN}

En poblaciones caucásicas, la fibrosis quística (FQ) presenta una tasa de portadores sanos estimada en 1 por cada 25 individuos ${ }^{30}$. En Chile, se había estimado una tasa de 1 en $40^{9}$. Conforme a la tasa calculada para Chile, se esperaba en este trabajo, encontrar solo un individuo portador de delta F508, entre los 50 individuos normales de la ciudad de Valparaíso. Sin embargo, se encontraron dos individuos portadores heterocigotos para dicha mutación. Por lo tanto, los resultados obtenidos indicarían que la tasa de portadores observada en la ciudad de Valparaíso es de 1 en 25 individuos, similar a la tasa descrita para poblaciones de origen europeo ${ }^{30}$.

El intervalo de confianza calculado para el tamaño de muestra analizado es de $85,77 \%$. Este intervalo de confianza es bueno y la varianza de la
Tabla 2. Frecuencias alélicas, genotípicas y haplotípicas de los polimorfismos de nucleótido único (SN Ps) en los 100 cromosomas analizados (50 individuos)

\begin{tabular}{|c|c|c|c|}
\hline SNP & $\begin{array}{c}\text { Alelo, } \\
\text { genotipo } \\
\text { o haplotipo }\end{array}$ & $\begin{array}{c}\text { Frecuencia } \\
\text { observada } \\
(\%)\end{array}$ & $\begin{array}{c}\text { Frecuencia } \\
\text { esperada } \\
(\%)\end{array}$ \\
\hline \multirow{5}{*}{ M470V } & Alelo 1 & 46 & - \\
\hline & Alelo 2 & 54 & - \\
\hline & Genotipo 1/1 & 24 & $21^{*}$ \\
\hline & Genotipo 1/2 & 44 & $50^{*}$ \\
\hline & Genotipo 2/2 & 32 & $29^{*}$ \\
\hline \multirow{5}{*}{ T854T } & Alelo 1 & 62 & - \\
\hline & Alelo 2 & 38 & - \\
\hline & Genotipo 1/1 & 42 & $38^{*}$ \\
\hline & Genotipo 1/2 & 40 & $48^{*}$ \\
\hline & Genotipo 2/2 & 18 & $14^{*}$ \\
\hline \multirow{4}{*}{ Haplotipos } & $2-1$ & 45 & $33,5^{\&}$ \\
\hline & $1-2^{\#}$ & 29 & $17,5^{\&}$ \\
\hline & $1-1$ & 17 & $28,5^{\&}$ \\
\hline & $2-2$ & 9 & $20,5^{\&}$ \\
\hline
\end{tabular}

Las frecuencias se presentan en porcentajes: El cálculo de las frecuencias esperadas no corresponde. ${ }^{*}$ Calculada según Levene ${ }^{34}$. \&Frecuencia esperada, asumiendo ausencia de ligamiento. \#Los dos cromosomas portadores de la mutación FQ delta F508 se encontraron dentro del mismo marco haplotípico.

frecuencia es baja, por lo que es altamente probable que la tasa de portadores se mantenga, aun aumentando el tamaño de la muestra. Esta frecuencia es representativa de los 229 individuos muestreados, y extrapolable a la población escolar de octavo básico de la comuna. La población escolar de octavo básico incluye todos los estratos socioeconómicos de la población total de la comuna de Valparaíso. Por lo tanto, los resultados reportados en este estudio son representativos de esta población. Sin embargo, las diferencias étnicas de las distintas regiones de Chile, no permite extrapolar estos resultados a la población chilena en general. Se pretende, en un estudio posterior, aumentar el tamaño muestral a 170 individuos (95\% de intervalo de confianza), extender el 
estudio a otras regiones del país e incorporar la detección de otras mutaciones presentes en la población chilena afectada de $\mathrm{FQ}^{20-22}$.

La frecuencia de portadores encontrada en este estudio, permite confirmar que la FQ en efecto está siendo subdiagnosticada. El grado de subdiagnóstico en la comuna de Valparaíso, y probablemente en el resto del país, puede ser aún mayor que el esperado basado en la incidencia calculada 9 .

De acuerdo a los resultados obtenidos mediante el cálculo de las frecuencias genotípicas esperadas, no se observa desviación del equilibrio Hardy-Weinberg, para los loci de ambos SNPs ni para el locus de la mutación delta F508 en esta muestra de la población de Valparaíso. La ausencia de desviación, implica que estas variantes no son nuevas, y que los eventos que las incorporaron en esta población (mutación o migración), se han diluido en el tiempo.

En el caso de Valparaíso, por tener una condición de puerto y haber sido uno de los más importantes en el siglo pasado, fue una de las principales puertas de entrada para los colonos europeos, a inicios del siglo XIX ${ }^{31}$. Estos inmigrantes, podrían haber traído la mutación delta F508, e introducirla en nuestra población. Sin embargo, la muestra poblacional utilizada presenta un porcentaje de mezcla amerindia de 51\%, calculado por la frecuencia de Rh negativo. Este porcentaje es mayor que el porcentaje de mezcla calculado para la población de Santiago $(35 \% 32)$. El porcentaje de mezcla caucásico-amerindio de la población de Santiago es la base para el cálculo de la incidencia de FQ a nivel nacional ${ }^{9}$. La muestra analizada en este estudio no presenta un aporte caucásico mayor que el observado en la población de Santiago, por lo tanto la mayor frecuencia de portadores encontrada no se explica solamente sobre la base del aporte de este grupo étnico.

Una posible explicación radica en la hipótesis basada en la selección natural llamada "ventaja del heterocigoto". En el caso de la FQ, los heterocigotos portadores de mutaciones en el gen CFTR tendrían una ventaja selectiva ${ }^{33}$. Una selección positiva de, aproximadamente, $2 \%$ sería suficiente para mantener la frecuencia de FQ en los niveles actuales ${ }^{33}$. La selección positiva de los heterocigotos podría explicar la no desviación del equilibrio de Hardy-Weinberg y la tasa de portadores observada, aun en presencia de mortalidad y fertilidad reducida de los homocigotos recesivos.

Por otra parte, se ha establecido, que la determinación de haplotipos informativos podría facilitar la búsqueda de mutaciones FQ dentro de una población y conocer su historia evolutiva. La mutación delta F508 y los SNPs M470V y T854T están en desequilibrio de ligamiento ${ }^{14}$. En los 100 cromosomas analizados, el haplotipo 2-1 resultó ser el más frecuente en la población general (45\%). Los dos portadores de la mutación delta F508 observados en este estudio, presentaron el mismo marco haplotípico (1-2) para dicha mutación. Este haplotipo también fue el segundo más frecuente entre los cromosomas normales (29\%). La recombinación que dio origen a este marco haplotípico, es más frecuente en países mediterráneos $(2,8 \%$ de los cromosomas portadores de delta F508 del sur de Francia ${ }^{25}$ ), que en el norte de Europa ${ }^{14,16}$. Consecuentemente a lo mencionado y dado que la mayoría de los inmigrantes que poblaron la ciudad de Valparaíso provenían desde España ${ }^{8,31}$, no es extraño que los cromosomas portadores de la mutación delta F508 encontrados en este estudio, presenten este marco haplotípico.

Sin embargo, el haplotipo para uno de los portadores se reconstruyó mediante inferencia Bayesiana. Es, por lo tanto, posible que el segundo cromosoma portador tenga un haplotipo asociado 1-1, que es el haplotipo encontrado con mayor frecuencia asociado a dicha mutación ${ }^{14,25}$.

La importancia de este trabajo radica, principalmente, en que es el primer estudio por muestreo realizado en Chile sobre la frecuencia de portadores de fibrosis quística, en población general. Además, ha permitido conocer la prevalencia de la mutación FQ más importante en este grupo de estudio, y estructurar una base de datos, para posteriormente realizar comparaciones con grupos de pacientes FQ típicos y atípicos.

Se hace evidente la necesidad de ampliar este tipo de estudio a otras ciudades del país, y complementarlo mediante el análisis de otros polimorfismos del gen CFTR. De esta manera, se tendrá una mayor información sobre la manifestación y prevalencia de esta enfermedad en la población chilena. 


\section{REFERENCIAS}

1. Mckusick-Nathans. Online Mendelian Inheritance in Man, OMIM (TM). Institute for Genetic Medicine, Johns Hopkins University (Baltimore, MD) and National Center for Biotechnology Information, National Library of Medicine (Bethesda, MD), 2000. World Wide Web URL: http:// www.ncbi.nlm.nih.gov/omim/

2. Rordan J, Rommens J, Kerem B, Alon N, Rozmahel R, GrZeiEZAK Z ET AL. Identification of the cystic fibrosis gene: cloning and characterization of complementary DNA. Science 1989; 245: 1066-73.

3. Schwiebert EM, Egan ME, Hwang T-H, Fulmer SB, Aluen SS, Cutting GR et aL. CFTR regulates outwardly rectifying chloride channels through an autocrine mechanism involving ATP. Cell 1995; 81: 1063-73.

4. Wine JJ. The genesis of cystic fibrosis lung disease. J Clin Invest 1999; 103: 309-12.

5. Bobadila JL, Macek M, Jr Fine JP, Farrell PM. Cystic fibrosis: a worldwide analysis of CFTR mutations correlation with incidence data and application to screening. Hum Mutat 2002; 19: 575-606.

6. Kerem B, Rommens J, Buchanan J, Markiewicz D, Cox T, ChaKRAVARTI A ET AL. Identification of the Cystic Fibrosis gene. Genetic analysis. Science 1989; 245: 1073-80.

7. Cystic Fibrosis Genetics Analysis Consortium. Cystic fibrosis mutation database 2004. World Wide Web URL: http://www.genet.sickkids.on.ca/cftr/

8. CRUz-Coke R. Origen y evolución étnica de la población chilena. Rev Méd Chile 1976; 104: 365-8.

9. Valenzueia C. Genética de la fibrosis quística. Rev Chil Pediatr 1988; 31: 218-9.

10. Sánchez I, Pérez MA, Boza ML, Lezana V, Vila MA, Repetto G et al. Consenso nacional de fibrosis quística. Rev Chil Pediatr 2001; 72: 356-80.

11. Cuppens H, Lin W, Joopers M, Costes B, Teng H, VanKeERBerghen A et al. Polyvariant Mutant Cystic Fibrosis Transmembrane Conductance Regulator Genes: The Polymorphic (TG)m locus explains the partial penetrance of the T5 Polymorphism as a Disease Mutation. J Clin Invest 1998; 101: 48796.

12. Steiner B, Galatti S, Schalefr A, Sanz J, Truninger K. Common CFTR gene polymorphism are associated with aberrant splicing in patients with chronic pancreatitis. Pancreatology 2002; 3: 209-69.
13. Lee JH, Choi JH, Namkung W, Hanraham J, Chang J, SONG SY ET AL. A haplotype-based molecular analysis of CFTR mutations associated with respiratory and pancreatic diseases. Hum Mol Genet 2003; 12: 2321-32.

14. Dörk T, Neumann T, Wulbrand U, Wulf B, Kalin N, MAAss G. Intra and extragenic marker haplotypes of CFTR mutations in cystic fibrosis families. Hum Genet 1992; 88: 417-25.

15. Morral N, Dörk T, Levadot R, Dziadek V, Mergier B. Haplotype analysis of 94 Cystic Fibrosis Mutations with seven polymorphic CFTR DNA markers. Hum Mutat 1996; 8: 149-59.

16. Cuppens H, Teng H, Raeymaekers P, De Boeck C, Cassiman J. CFTR haplotype background on normal and mutant CFTR genes. Hum Mol Genet 1994; 3: 607-14.

17. BertranPetit J, CALAFEL F. Genetic and geographical variability in cystic fibrosis: evolutionary considerations. En: Cardew G (ed) Variation in the human genome. Chichester, Wiley \& Sons, 1996; 97-118.

18. Mateu E, Calafell F, Ramos MD, Casals T, BertranPETIT J. Can a place of origin of the main cystic fibrosis mutations be identified? Am J Hum Genet 2002; 70: 257-64.

19. L W, Eueswort D, Krushral J, Chang B, HewettEMMETT D. Rates of nucleotide substitution in primates and rodents and the generation time effect hipothesis. Mol Phylogenet Evol 1996; 5: 182-7.

20. Ríos J, Oreluana O, Aspilaga M, Avendano I, Largo I, Riveros N. CFTR mutations in Chilean cystic fibrosis patients. Hum Genet 1994; 94: 291-4.

21. Repetto G, Poggi H, Harris P, Navarro H, Sánchez I, GuiraLdes E. Identificación de mutaciones en el gen CFTR en pacientes chilenos con fibrosis quística. Rev Méd Chile 2001; 129: 841-7.

22. Molina G, González F, Cave R, Cornejo M, Navarro $\mathrm{S}$, Degun M et al. Estudio clínico-genético molecular de la fibrosis quística en la $\mathrm{V}$ región, Chile. Rev Méd Chile 2002; 130: 850-8.

23. WeIR B. Within Population Variance of Allele Frequencies. En: Genetic Data Analysis II. Sunderland, Sinauer Associated, Inc. 1996; 37-40.

24. Zieiensky J, Rozmahel R, Bozon D, Kerem B, GrzelcZaK Z, RIoRDAN J Et AL. Genomic DNA sequence of the Cystic Fibrosis Transmembrane Conductance Regulator (CFTR) gene. Genomics 1991; 10: 214-28.

25. Claustres M, Desgeorges M, Moine P, Morral N, EsTrviL X. CFTR haplotypic variability for normal and mutant genes in cystic fibrosis families from southern France. Hum Genet 1996; 98: 336-44. 
26. MiLER S, DYKES D. A simple salting out procedure for extracting DNA from human nucleated cells. Nucleic Acids Res 1988; 16: 1215.

27. Friedman K, Highsmith W, Silverman L Detecting multiple cystic fibrosis mutations by polymerase chain reaction-mediated site-directed mutagenesis. Clin Chem 1991; 37: 753-5.

28. LEVENE H. On a matching problem in genetics. Ann Math Stat 1949; 20: 91-4.

29. Niu T, Q in ZS, Xu X, Lu JS. Bayesian haplotype inference for multiple linked single-nucleotide polymorphisms. Am J Hum Genet 2002; 70: 157-69.
30. Rosenstein B, Langbaum T. Diagnosis in cystic fibrosis. Taussing LM, eds. New York, ThiemeStratton, Inc, 1984; 87-114.

31. Estrada B. Valparaíso. Sociedad y Economía Siglo $\mathrm{XX}$. Serie monografías históricas № 12, Instituto de Historia, Universidad Católica de Valparaíso, Ediciones de Valparaíso 2000.

32. Cifuentes L, Valenzuela CY, Cruz-Coke R, Armanet L, Lyng C, HaRB Z. Caracterización genética de la población hospitalaria de Santiago. Rev Méd Chile 1988; 116: 28-33.

33. Rodman DM, Zamudio S. The cystic fibrosis heterozygote advantage in surviving cholera? Med Hypotheses 1991; 36: 253-8.

Agradecimientos:

Se agradece a los directores, profesores, apoderados y especialmente a los alumnos de los siguientes establecimientos: Colegio Agustín Edwards, Colegio Matilde Brandeau de Rosas, Escuela Básica Gaspar Cabrales, Escuela Básica Alemania, Escuela Básica Pacífico, Liceo de Niñas Juana Ross de Edwards, Escuela Básica Blas Cuevas-Ramón Allende, Colegio Luterano Concordia, Liceo Hernán Olguín, Escuela Básica República de Paraguay, Liceo №1 de Niñas María Franck, Escuela Básica República de Bolivia, Liceo Carlos Cousiño, Escuela Particular Santa Ana, Escuela Básica Carabinero Pedro Carriaga M, Liceo Co-educacional La Igualdad, Colegio Salesiano de Valparaíso, Scuola Italiana Arturo Dell'Oro, Liceo Politécnico Computacional A. Nazar, Colegio Arturo Edwards, Escuela Básica Grecia, Colegio Alberto Hurtado II, Colegio Particular Adventista, Colegio Cecal, Escuela Básica República de Argentina, Escuela Básica Cirujano Videla, Escuela Básica República del Salvador, Escuela Básica Federico Albert, Escuela Básica América, Escuela Lorenzo Luzuriaga, Escuela Básica Árabe Siria, Escuela Básica Ciudad de Berlín, Colegio Internacional.

En memoria de la señora Silvia Grez Casarino, cuya intercesión permitió formar este grupo de trabajo. 